\title{
Energy-dissipative dual-crosslinked hydrogels for dynamically super-tough sensors
}

\author{
Funian $\mathrm{Mo}^{1,2}$, Zifeng Wang ${ }^{2}$, Ruijuan Jiang ${ }^{3 *}$, Weiming $\mathrm{Gai}^{3}$, Qing $\mathrm{Li}^{2}$, Shuangkun $\mathrm{Lv}^{4}$ and \\ Chunyi Zhi ${ }^{2 *}$
}

\begin{abstract}
In the fields of electronic skin and soft wearable sensors, intrinsically stretchable conductors undergo rapid development; however, practical applications of artificial skinlike materials/devices have not been realized because of the difficulty in combining the electromechanical properties and sensing performance. Contrarily, insoluble inorganic conductive domains in the hydrogel matrix are generally incompatible with surrounding elastic networks, decreasing the mechanical strength. Usually, the hydrogels are vulnerable either to severe mechanical stimuli or large deformation, especially when notches are induced. In this study, based on an energy-dissipative dual-crosslinked conductive hydrogel, a mechanically durable and super-tough strain sensor was developed. The highly soft yet dynamically tough hydrogel demonstrated high ionic conductivity $\left(30.2 \mathrm{mS} \mathrm{cm}^{-1}\right)$, ultrastretchability ( $>600 \%$ strain), and superior linear dependence of strain sensitivity with a maximum gauge factor of 1.2 at $500 \%$ strain. Because of these advantageous synergistic effects, the resultant hydrogel strain sensor demonstrated reliable and stable detection of a large range of human motion and subtle vibrations. Moreover, it impressively exhibited super toughness that could endure consecutive treading pressure and even retain normal operation after 20 times of car run-over on the road. These demonstrations highly confirm the sensor's superior mechanical durability and reliability, displaying great potential in developing next-generation mechanically adaptable sensors.
\end{abstract}

Keywords: energy-dissipative, hydrogels, super-tough, wearable, strain sensors

\section{INTRODUCTION}

Artificial skin-like conductive materials, which have been extensively used as strain sensors to convert external mechanical stimuli into electrical signals, have attracted considerable interest in the fields of electronic skins [1-4], artificial intelligence [5], human motion detection $[6,7]$, and physiological monitoring [8,9]. However, for traditional hydrogel conductors blended with electronically conductive fillers such as rigid conjugated polymers [10], conductive inorganic nanoparticles [11,12], or liquid metals [13], the compatibility between conductive domains and surrounding insulating elastic networks in the gel matrix remains unsatisfactory, which results in poor electromechanical performance and decay of mechanical strength. Recently, ionic hydrogel conductors, which exhibit better affinity to polymeric networks, have been applied as ionically conductive components such as water-soluble metallic salts $[14,15]$ and ionic liquids [16-18]. Unlike electronically conductive fillers, salt solutions/ionic liquids in the ionic conductor are dominant as solvents of the hydrogel matrix with the polymeric chains embedded in them, which can guarantee the continuity of the conductive phase in arbitrary deformations.

Considerable efforts have been dedicated to improving the durability and serviceability of flexible conductive materials, such as integrating self-healability and stretchability into rigid conjugated polymers to endow them with the capability of mending notches and deformation adaptability $[15,19]$. Unfortunately, self-healing is a passive strategy that normally requires meticulous

\footnotetext{
${ }^{1}$ Flexible Printed Electronics Technology Center, Harbin Institute of Technology (Shenzhen), Shenzhen 518055, China

${ }^{2}$ Department of Materials Science and Engineering, City University of Hong Kong, Hong Kong 999077, China

${ }^{3}$ Shenzhen Municipal Engineering Design \& Research Institute Co., Ltd, Shenzhen 518055, China

${ }^{4}$ Songshan Lake Materials Laboratory, Dongguan 523808, China

* Corresponding authors (emails: jiangrj@hotmail.com (Jiang R); cy.zhi@cityu.edu.hk (Zhi C))
} 
manual alignment of broken pieces; in certain cases, external stimuli are required to trigger the healing process. Moreover, electromechanical properties are hardly well preserved, which deteriorates the device's performance. Furthermore, although stretchability can make devices accommodate mechanical deformations, most general stretchable hydrogels are extremely notch-sensitive, indicating that once substantial notches or cracks are induced during stretching, the mechanical strength would seriously decay, leading to eventual fracture $[20,21]$. Overall, neither of the above strategies can thoroughly satisfy the requirements for practical wearable applications because these designs only tackle the "flexibility" issue ostensibly but cannot provide enough mechanical softness, fracture strength, and dynamic stability for flexible conductors.

Herein, by employing an energy-dissipative dualcrosslinked hydrogel conductor, a highly flexible yet dynamically super-tough strain sensor was developed. The hydrogel composed a typical interpenetrating network structure of covalently crosslinked polyacrylamide (PAM) and $\mathrm{Al}^{3+}$-crosslinked alginate. In the latter network, the guluronic acid units on the molecular alginate chains formed abundant ionic crosslinks with surrounding dissociated $\mathrm{Al}^{3+}$, which could dynamically dissipate energy during deformation by the dissociation of physical bonding on stress loading and recovery while unloading $[22,23]$. Moreover, the hydrogel possessed excellent conductivity in the presence of dissolved $\mathrm{Li}_{2} \mathrm{SO}_{4}$, which enabled the assembled hydrogel sensor with high sensitivity (gauge factor (GF) $>1.2$ at 500\% stretch strain) and stable resistance variation on severe deformations and mechanical impacts such as being dynamically stretched, violently hammered, and heavily compressed. Furthermore, for quantitative human motion detection, the excellent electromechanical stability of the dual-crosslinked hydrogel made it extremely suitable as a reliable and wearable sensor. Despite a catastrophic impact subsequent to being run over by 20 cars, the hydrogel sensor could well maintain its elasticity and sensitivity without malfunction, thus confirming its superior structural integrity and deformation adaptability.

\section{EXPERIMENTAL SECTION}

\section{Preparation of Al-alginate-based super-tough hydrogel}

Super-tough hydrogels were synthesized using acrylamide (AM, AR grade, Aladdin) as the monomer, ammonium persulphate (APS) (>98\%, Acros Organics) as the radical initiator, $N, N^{\prime}$-methylenebisacrylamide (MBAA) (99\%, Aladdin) as the crosslinker, and $N, N, N^{\prime}, N^{\prime}$-tetramethylethylenedia-mine (TEMED) (99\%, Aladdin) as the crosslinking accelerator. The mixture containing $0.1 \mathrm{~mol} \mathrm{~L}^{-1} \mathrm{AlCl}_{3}$ (99\%, Alfa Aesar) and $1 \mathrm{~mol} \mathrm{~L}^{-1} \mathrm{Li}_{2} \mathrm{SO}_{4}$ (99.7\%, Alfa Aesar) was prepared as the solvent before polymerization.

The $\mathrm{Al}^{3+}$-crosslinked alginate/PAM (Al-alginate/PAM) hydrogel electrolyte was synthesized via two steps. First, $3.0 \mathrm{~g}$ AM monomer was dissolved in $37 \mathrm{~mL}$ deionized water, followed by adding $24.0 \mathrm{mg}$ APS and $3.5 \mathrm{mg}$ MBAA as the initiator and crosslinker, respectively. After stirring for $20 \mathrm{~min}, 0.72 \mathrm{~g}$ alginate and two drops of TEMED were added to the abovementioned solution and vigorously stirred overnight until complete dissolution. To allow polymerization, after degassing by vacuum, the resultant solution was poured into a glass mold and placed in an oven at $65^{\circ} \mathrm{C}$ for $2 \mathrm{~h}$ to obtain the alginate/ PAM hydrogel. Second, to allow ion exchange, the resulting alginate/PAM hydrogel was immersed in the mixture of $0.1 \mathrm{~mol} \mathrm{~L}^{-1} \mathrm{AlCl}_{3}$ and some amount of $\mathrm{Li}_{2} \mathrm{SO}_{4}$ for $1 \mathrm{~h}$. During this step, ionically crosslinked alginate networks were formed through the interaction with $\mathrm{Al}^{3+}$. The comparative PAM hydrogel electrolyte was similarly prepared without adding alginate.

\section{Assembly of the strain sensor}

The strain sensor was assembled with the hydrogel serving as an ionic conductor and $4910 \mathrm{VHB}$ elastomer tape as substrates. To prevent the evaporation of the hydrogel moisture, the top and bottom of the strain sensor were connected with $\mathrm{Cu}$ foil, which served as the electrodes, and covered with $4910 \mathrm{VHB}$ tapes.

\section{Material characterization}

Thermogravimetric analysis (TGA) was conducted using the TA Instrument TG Q50 system in the temperature range of $30-600^{\circ} \mathrm{C}$ with a heating rate of $10^{\circ} \mathrm{C} \mathrm{min}$. Fourier transform infrared (FTIR) spectroscopy was conducted using a Perkin Elmer Spotlight 400 Microscopy System in the range of $450-4000 \mathrm{~cm}^{-1}$. Before characterization, the hydrogel samples were freeze-dried, crushed, and mixed with $\mathrm{KBr}$ to yield pellets. Tensile tests were conducted using the HTSLLY9130A tensile machine (Guangdong Zhongye Instrument Equipment Co., Ltd.). The hydrogel samples were first cut into dumbbell-like shapes with $40.0 \mathrm{~mm}$ length and $20.0 \mathrm{~mm}$ width. During the tensile test, the piston velocity during measurement was set as $15 \mathrm{~mm} \mathrm{~min}^{-1}$. The tensile modulus was de- 
termined as per the average slope in the strain range of $20 \%-60 \%$ based on the stress-strain curve. The hydrogel samples' morphologies were characterized using an environmental scanning electron microscope (ESEM, FEI/ Philips XL30).

\section{Calculation method of fracture energy}

The fracture energy of the hydrogels was calculated as per a method reported by Sun et al. [24] and Rivlin et al. [25]. In particular, two samples $(40.0 \mathrm{~mm} \times 20.0 \mathrm{~mm} \times$ $2.0 \mathrm{~mm}$ ) of the same hydrogel were pulled with a tensile machine, of which one sample was notched by scissors (a $10.0 \mathrm{~mm}$-long notch) while the other was intact without a notch. Thus, force-distance curves were obtained by pulling these two hydrogel samples at a piston velocity of $20 \mathrm{~mm} \mathrm{~min}^{-1}$, and the integrated area under the curve represents the work performed by the applied force, $U(L)$. Upon pulling, the notch gradually turned into a running crack, and the fracture energy was calculated as follows

$\left.\Gamma=U\left(L_{\mathrm{c}}\right)\right) /\left(a_{0} \times b_{0}\right)$,

where $L_{c}$ is the critical distance when pulling the notched sample from the original position to the place when a running crack occurred, and $U\left(L_{\mathrm{c}}\right)$ is the integrated area under the force-length curve of the unnotched sample at the distance of $L_{c}, a_{0}$ and $b_{0}$ are the initial width and thickness of samples, respectively.

\section{Electrochemical measurements}

The ionic conductivity of the polymer electrolyte was calculated by ohmic resistance, which was obtained from the alternating current (AC) impedance spectra that were tested by an electrochemical workstation (CHI 760D). The ionic conductivity is determined as follows:

$\sigma=l / R A$,

where $\sigma$ is the ionic conductivity of hydrogels, and $l, R$, and $A$ represent the thickness, bulk resistance, and test area of hydrogels, respectively.

The electrical resistance of hydrogels was tested by a Keithley analyzer (Model 6487). The relative resistance changes of hydrogels were calculated as follows $[7,26]$ :

$\Delta R / R_{0}=\left(R-R_{0}\right) / R_{0} \times 100 \%$,

where $R_{0}$ is the resistance without strain and $R$ represents the real-time resistance.

The sensory performance of the hydrogel sensor was estimated by the GF, which is defined as the relative change in resistance with respect to stretch strain:

$\mathrm{GF}=\left(\left(\Delta R / R_{0}\right) /\left(\left(\Delta l / l_{0}\right)\right.\right.$,

where $R_{0}$ is the resistance at $0 \%$ strain and $R$ is the re- sistance under stretching deformation.

\section{RESULTS AND DISCUSSION}

\section{Formulation and characterization of Al-alginate-based super-tough hydrogel}

The Al-alginate/PAM hydrogel comprises a typical dualcrosslinked network structure (Fig. 1a). The first framework is formed by a covalently crosslinked PAM network with alginate chains interacting in the matrix through both physical entanglement and intertwining. Subsequently, alginate units form the second network through ionic crosslinks by $\mathrm{Al}^{3+}$. Furthermore, the synthesis route (Fig. 1b) is divided into two steps. First, the AM monomer aqueous solution containing dissolved sodium alginate was polymerized to form a hydrogel through free radical polymerization. The covalent crosslinks in the PAM network were formed by the dual-double bonds from MBAA crosslinkers. Next, the as-prepared hydrogel was immersed in an aqueous solution containing $0.1 \mathrm{~mol} \mathrm{~L}^{-1} \mathrm{AlCl}_{3}$ and various $\mathrm{Li}_{2} \mathrm{SO}_{4}$ contents until complete ion exchange to finally obtain the transparent Al-alginate/PAM hydrogel (Fig. S1a). In this process, the dissociated $\mathrm{Al}^{3+}$ contacted the guluronic acid units of molecular alginate chains and formed abundant ionic crosslinks. Thus, an interpenetrating network within the hydrogel matrix was generated. Moreover, the absorbed $\mathrm{Li}_{2} \mathrm{SO}_{4}$ was responsible for the ionic conductivity for sensor operation.

The energy dissipation mechanism of the super-tough Al-alginate/PAM hydrogel could be interpreted as follows: on stress loading, the dynamic ionic crosslinks separate and dissipate energy such that the covalently crosslinked PAM network remains intact, preventing the strength and shape collapse of the hydrogel. Upon stress unloading, ionic crosslinks reform, and the PAM network recovers to a relaxation state, leading to hydrogel recovery. The hydrogel structure was characterized by FTIR, which indicated that the PAM and alginate matrix exhibited independent structures, which revealed the physical interpenetrating network (Fig. S1b). TGA test was performed on the hydrogel conductor within the temperature range of $30-600^{\circ} \mathrm{C}$. The result indicated that the hydrogel's moisture content gradually evaporated from the hydrogel matrix, and the residue content at $600^{\circ} \mathrm{C}$ revealed that the entire solid content inside the hydrogel conductor was $\sim 65 \%$, which was obtained according to the residual weight ratio at $150^{\circ} \mathrm{C}$ (Fig. S2).

The mechanical properties of the pure PAM hydrogel, non-ionically crosslinked alginate/PAM hydrogel, and Al- 

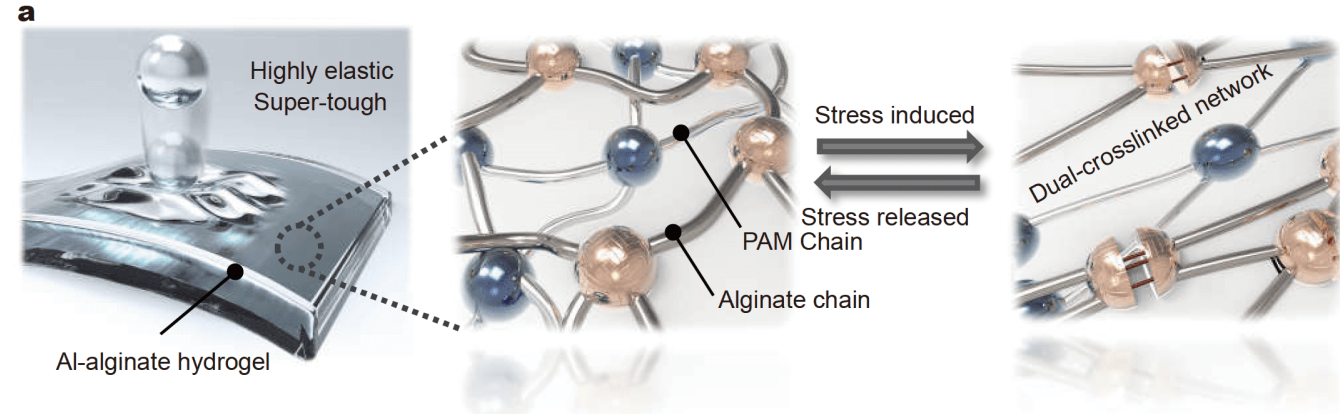

b

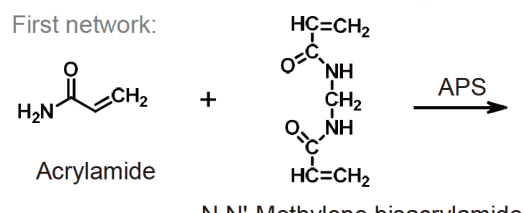

N,N'-Methylene bisacrylamide

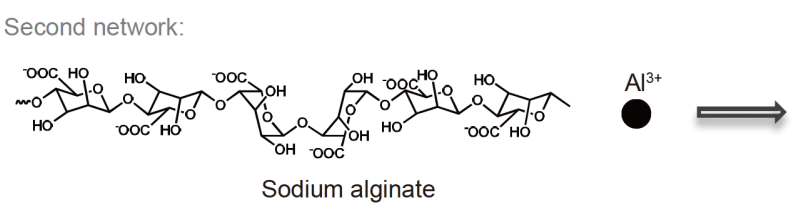

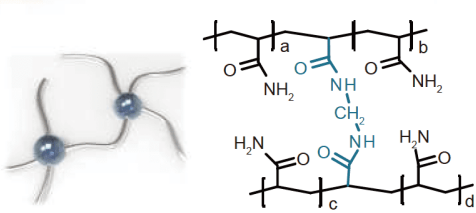

Covalent crosslink

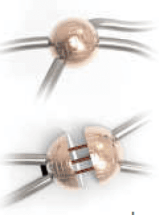

lonic crosslink

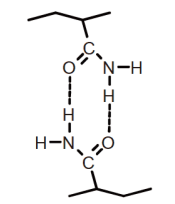

Hydrogen bonding

Figure 1 (a) Schematic of the polymeric matrix of Al-alginate/PAM hydrogel with a dual-crosslinked network structure. (b) Synthesis route of Alalginate/PAM hydrogels and corresponding molecular structures of covalent and ionic crosslinks. Under stress loading, ionic crosslinks between $\mathrm{Al}^{3+}$ and alginate chains dissociate and dissipate energy. On stress relaxation, crosslinks are rebuilt to recover the hydrogel.

alginate/PAM hydrogel were systematically investigated by fracture toughness and tensile tests. First, fracture toughness measurement was performed on different hydrogels with and without ionic crosslinks (Fig. 2a). A notch was cut on one edge of the hydrogel. Next, the hydrogels were stretched with a tensile testing machine till notch propagation. Fig. $2 b$ shows the comparison of the calculated fracture energies of different hydrogels, and the detailed description of the fracture energy determination is shown in the EXPERIMENTAL SECTION. The results indicate that the nonionic-crosslinked pure PAM and alginate/PAM hydrogels exhibit comparative fracture toughness of 0.397 and $0.442 \mathrm{~kJ} \mathrm{~m}^{-2}$, respectively, while the ionically crosslinked Al-alginate/PAM hydrogel displays an ultra-high fracture toughness of $9.621 \mathrm{~kJ} \mathrm{~m}^{-2}$, outperforming other substantial hydrogel materials (Fig. S3). This significant enhancement in fracture toughness can be interpreted using the Lake-Thomas model $[27,28]$. For the nonionic-crosslinked network of PAM and alginate/PAM hydrogels, the stress concentration occurs around the surrounding gel matrix adjacent to the notch when the notch is stretched. Thus, once the notch turns into a running crack, localized damage first occurs in the adjacent covalent polymeric chains and dissipates the stored energy, resulting in small fracture energy. For the Al-alginate/PAM hydrogel, the ionically crosslinked network bridges the covalent network and can be reversibly unzipped over a large region under an external force, which effectively alleviates the concentrated stress on the PAM covalent network and stabilizes deformation, which increases the fracture toughness.

Second, the tensile test results indicated that the elastic modulus of the PAM-based hydrogel significantly increased from 2.85 to $453.4 \mathrm{kPa}$ after introducing the $\mathrm{Al}^{3+}$ crosslinked alginate network, which highly demonstrates the reinforcing effect of dual-crosslinked structural design on mechanical strength (Fig. 2c). Moreover, the toughened hydrogel became less stretchable because of the increased modulus derived from the limited ductility of the dual-crosslinked network; however, it was still rubbery and soft enough to be stretched by a tensile machine, approaching $600 \%$ (Fig. S4). With an increase in tensile velocity, the elongation-at-break of the Al-alginate/PAM hydrogel slightly decreased owing to the stress-strain hysteresis in the polymeric matrix during the fast- 
a
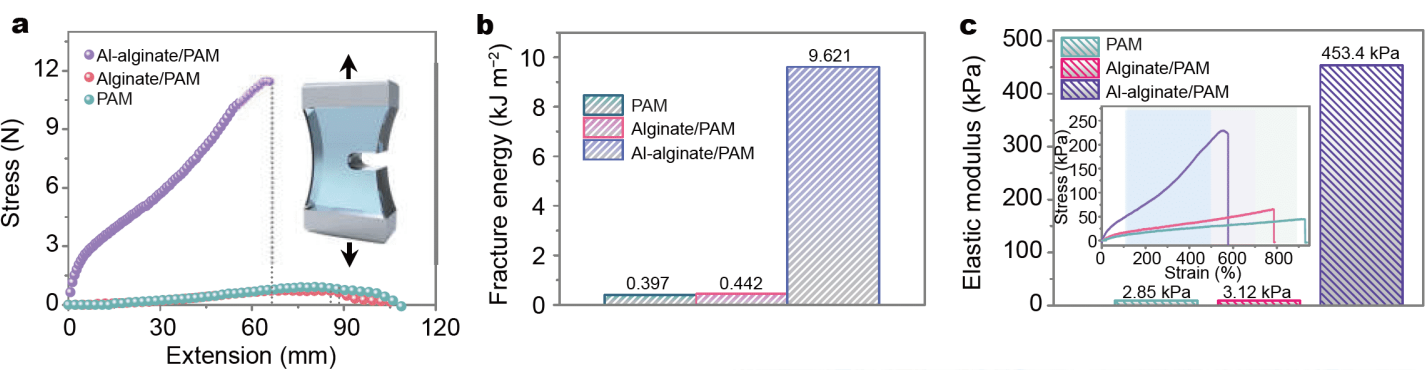

(
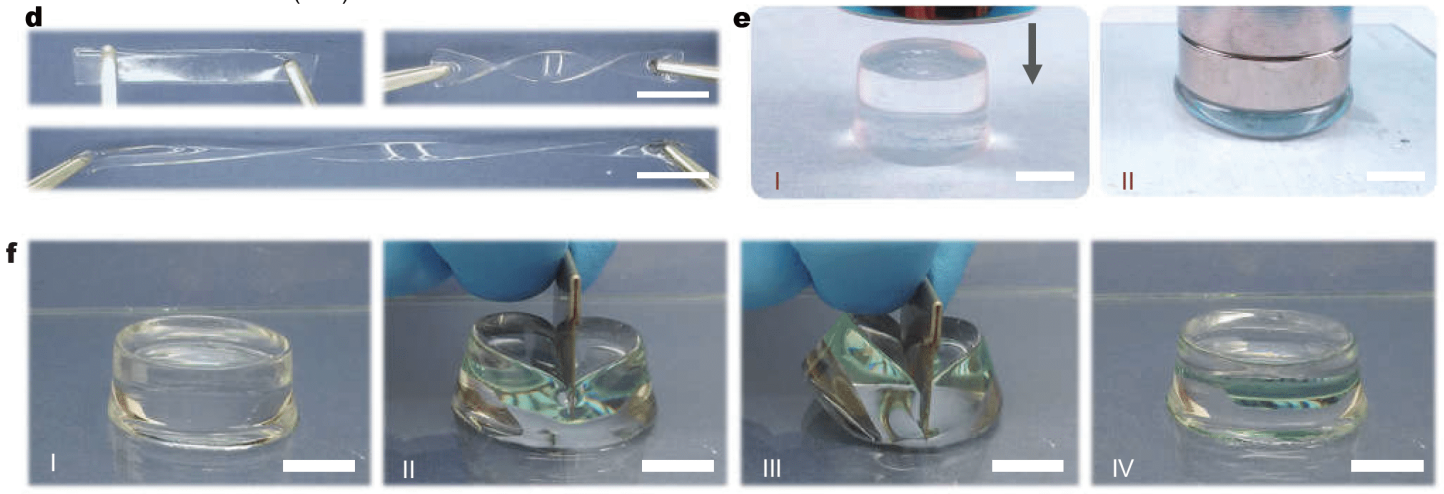

$\mathbf{g}$
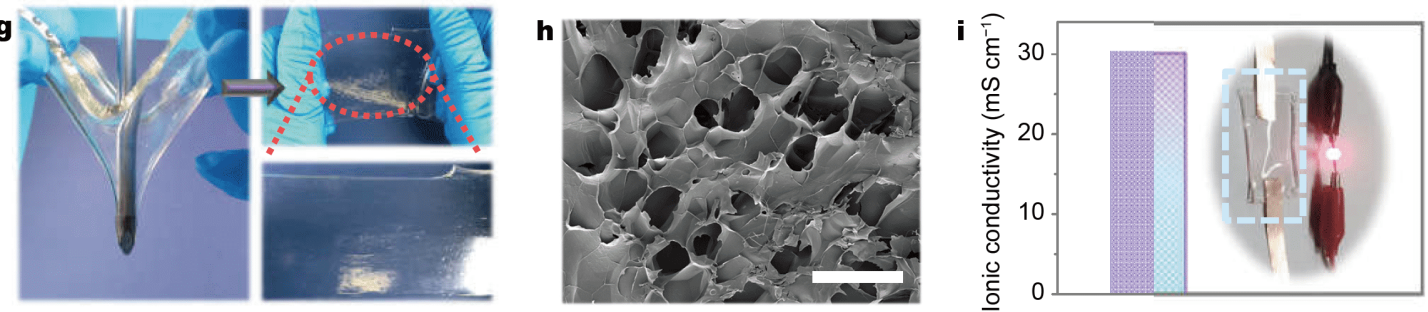

Figure 2 (a) Force-length profiles of PAM, alginate/PAM, and Al-alginate/PAM hydrogels. (b) Calculation of fracture energies of different hydrogels. (c) Tensile moduli of different hydrogels calculated from stress-strain curves. (d) Optical images of twisting and stretching an Al-alginate/ PAM hydrogel. Scale bar: $1 \mathrm{~cm}$. (e) Images of the compression of an Al-alginate/PAM hydrogel with heavy loading. Scale bar: $1 \mathrm{~cm}$. (f) Images of cutting an Al-alginate/PAM hydrogel with a sharp knife from top to bottom. Scale bar: $1 \mathrm{~cm}$. (g) Images of poking a stretched Al-alginate/PAM hydrogel with a cross screwdriver. (h) SEM image of a freeze-dried hydrogel, which reveals the interconnected porous structure. Scale bar: $10 \mu \mathrm{m}$. (i) Ionic conductivity of super-tough hydrogel containing $1.5 \mathrm{~mol} \mathrm{~L}^{-1} \mathrm{Li}_{2} \mathrm{SO}_{4}$. Inset: photograph showing the hydrogel film operating as an ionic conductor in an LED circuit.

stretching process (Fig. S4c). Fig. S5 shows that the storage modulus of the alginate/PAM hydrogels exhibited small changes after immersion in $\mathrm{Li}_{2} \mathrm{SO}_{4}$ solutions with various concentrations. A possible explanation is that although $\mathrm{Li}^{+}$could interact with guluronic acid units through the coordination bond, this bonding formed between the alginate chains and the monovalent ions could not form a 3D crosslinking network. Therefore, $\mathrm{Li}^{+}$ had limited effects on the mechanical properties of the hydrogel. Furthermore, the non-ionically crosslinked PAM and alginate/PAM hydrogels exhibited simple elastic behavior, whereas the Al-alginate/PAM displayed the two separated stages with a yield point during stretching (Fig. S4). A possible explanation is that, on the tensile test, the $\mathrm{Al}^{3+}$-crosslinked network first largely deformed and dominated the energy dissipation before yielding. Then, the $\mathrm{Al}^{3+} /$ alginate crosslinking points were separated, leading to slippage and orientation of molecular chains. Subsequently, the second chemically crosslinked PAM network started deforming largely. Therefore, the complete mechanical properties and behavior depended on the interactions of dual-crosslinked networks [29]. Fig. 2d shows that the Al-alginate/PAM hydrogel could be stretched to $100 \%$ strain under a twisting state, further revealing the energy dissipation ability.

Moreover, since both the concentration of the multivalent ion salt solution and the immersion time of hy- 
drogels in the solution are important factors for the ionic crosslinking of alginate, we systematically tested the various effects of $\mathrm{AlCl}_{3}$ on the ultimate interpenetrating network. The crosslinking effects are ascribed to the metal coordination of polyvalent metal ions and guluronic acid units on alginate chains. $\mathrm{Al}^{3+}$ could enable the alginate chains with a 3D crosslinking. Fig. S6a shows that by immersing alginate/PAM hydrogels in $0.1 \mathrm{~mol} \mathrm{~L}^{-1} \mathrm{AlCl}_{3}$ solution under different immersion times, the storage modulus of the tensile result increases owing to the gradual formation of the $\mathrm{Al}^{3+}$-crosslinked network. However, the elongation-at-break would gradually decrease because of the decline in molecular network scalability. By immersing the alginate/PAM hydrogels in $\mathrm{AlCl}_{3}$ solutions with various concentrations, the tensile strain of the ionically crosslinked hydrogels decreases when the $\mathrm{AlCl}_{3}$ concentration exceeds $0.5 \mathrm{~mol} \mathrm{~L}^{-1}$ (Fig. S6b), because excessive crosslinking adversely affects the extensibility of molecular chains in the interpenetrating hydrogel network. Moreover, the ionic conductivity of the Al-alginate/ PAM hydrogels after being immersed in different concentrations of $\mathrm{AlCl}_{3}$ solution was tested by AC impedance technology. The results indicate that as the concentration of $\mathrm{AlCl}_{3}$ solution increases, the calculated ionic conductivity of the Al-alginate/PAM hydrogels gradually decreases (Fig. S7) because although the increasing crosslinking degree by the $\mathrm{Al}^{3+}$ ionic crosslinking could enhance the mechanical robustness of the hydrogels, it increases the density of the microporous network of hydrogel matrix and adversely affects the electrolyte ion transportation, which decreases the ionic conductivity. Therefore, appropriate concentration and immersion time of $\mathrm{AlCl}_{3}$ for ionic crosslinking is essential to avoid excessive crosslinking.

A 2-cm-thick Al-alginate/PAM hydrogel was forcibly compressed to $\sim 33 \%$ of its initial height to demonstrate its mechanical advantages under severe deformations. The super-tough hydrogel could completely recover without any crack or partial rupture after unloading (Fig. 2e and Fig. S8). Moreover, an Al-alginate/PAM hydrogel was severely cut from top to bottom with a sharp blade, after which no visible cracks were observed (Fig. 2f). These results firmly certified the effective energy dissipation of the dual-crosslinked network. Under compressing/cutting states, the imposed stress on the Alalginate/PAM hydrogel could be largely dispersed by the $\mathrm{Al}$-alginate network through the reversible unzipping of sufficient $\mathrm{Al}^{3+}$ ionic crosslinks, which allows the covalently crosslinked PAM network to avoid stress concentration. Thus, the whole hydrogel matrix was stabilized. Furthermore, we adopted the pure PAM and non-crosslinked alginate/PAM hydrogels with the same water content as a comparison, and the same operations were performed on them. The comparative hydrogels showed relatively brittle characteristics that varying degrees of damages occurred on the hydrogels during compression and cutting (Figs S8 and S9). These hydrogels could not dissipate energy on deformation because of the lack of reversible ionic crosslinks as their covalent chains were directly exposed to concentrated stress, resulting in different molecular chains' rupture and gel matrix damage. Furthermore, a stretched Al-alginate/ PAM hydrogel could be severely poked by a sharp cross screwdriver (Fig. 2g). Because of the effective energy dissipation, the super-tough and mechanically flexible hydrogel could endure extremely high-concentrated stress and reverse to the initial state after the force unloading, confirming its superior toughness and dynamic stability.

SEM image in Fig. $2 \mathrm{~h}$ shows the interconnected microporous structure of the freeze-dried super-tough hydrogel that favored the filling and ion transport of sufficient protons. The calculated ionic conductivity of the super-tough hydrogel significantly increased with an increase in the concentration of the contents of dissolved $\mathrm{Li}_{2} \mathrm{SO}_{4}$ salts, which reached $>30.2 \mathrm{mS} \mathrm{cm}{ }^{-1}$ when the concentration of the contents exceeded $1.5 \mathrm{~mol} \mathrm{~L}^{-1}$. Finally, it approached a constant level of $31 \mathrm{mS} \mathrm{cm}^{-1}$ by continuously adding $\mathrm{Li}_{2} \mathrm{SO}_{4}$ to increase the concentration of the contents to $2.5 \mathrm{~mol} \mathrm{~L}^{-1}$ (Fig. S10a and b). Herein, the sample with $1.5 \mathrm{~mol} \mathrm{~L}^{-1} \mathrm{Li}_{2} \mathrm{SO}_{4}$ was selected as a model system for additional analysis. The ionic conductivity of the super-tough hydrogel was comparable to that of PAM and alginate/PAM hydrogels with equivalent $1.5 \mathrm{~mol} \mathrm{~L}^{-1} \mathrm{Li}_{2} \mathrm{SO}_{4}$ (Fig. S10c). For the slightly decreasing trend of the ionic conductivity from Al-alginate/PAM to alginate/PAM to pure PAM, a possible explanation is that as the crosslinking degree increases from pure PAM to alginate/PAM to Al-alginate/PAM hydrogel, the density of the microporous polymeric matrix increases, which may adversely affect the ion transportation of electrolyte ions in the hydrogel matrix. This result revealed that the formation of the dual-crosslinked structure only slightly affected ion transportation through the super-tough hydrogel. Before ionic crosslinking of alginate and $\mathrm{Al}^{3+}$ (Fig. S11a), $\mathrm{Li}_{2} \mathrm{SO}_{4}$ would be absorbed into the hydrogel without leakage during immersion and endowed the hydrogel with electrochemical properties (Fig. S11b). As a demonstration, the super-tough hydrogel could be connected in a circuit as an ionic conductor to light up a 
light-emitting diode (LED) (Fig. 2i). Moreover, we used the ultra-thin $\mathrm{Cu}$ foil as conductive electrodes for fabricating the strain sensors, and the hydrogel exhibited good adhesion to the electrodes' surface through hydrophilic interactions and hydrogen bonds' formation (Fig. S12). Furthermore, Fig. S13 shows that the Al-alginate/PAM hydrogel exhibits a higher moisture retention ability than the pure PAM hydrogel, possibly because the highly hydrated carboxyl groups on alginate chains could form abundant electrostatic interactions with water molecules, thus providing the water retention capacity of the Alalginate/PAM hydrogel.

\section{Characterizations of the mechanical properties of the super-tough hydrogel sensor}

Many studies have focused on the field of hydrogel-based sensors, and an ideal hydrogel conductor for sensory systems requires the synergistic effects of high mechanical properties and high ionic conductivity [30-33]. The synthesized super-tough ionic conductive hydrogels show high sensitivity and superior stability to diverse mechanical stimuli. As shown in Fig. 3a, the relative resistance changes of the hydrogel sensor exhibited a clear stepwise increasing trend as the strain increased from $0 \%$ to $200 \%$. After stress removal, the hydrogel sensor recovered to its original shape, and the resistance value returned to the initial level. Similarly, when the hydrogel was at a twisting state, the resistance variation was substantially consistent with strain during stretching, indicating good durability of its electromechanical property (Fig. 3b). Subsequently, Fig. 3c shows the response signals from the hydrogel sensor for different bending angles of $45^{\circ}, 90^{\circ}$, and $135^{\circ}$, in which the relative resistance changes increased as the bending angle increased. Moreover, constant electrical resistance could be maintained during the bending-holding process, showing the hydrogel sensor's high strain sensitivity and electrical stability.

Dynamically dramatic deformation processes of folding and squeezing, hammering, and compression were subsequently applied, and relative resistance responses upon stimulus were monitored in real time to simulate diverse mechanical impacts that would be confronted in real applications (Fig. 3d-f). The hydrogel sensor was robust enough to endure vastly arbitrary deformations because of various mechanical stimuli, and the imposed impact energy could be dissipated by the stable dual-crosslinked structure such that the sensing performance remained unaffected. These encouraging results indicate the promising potential of the super-tough hydrogels to be designed as fast-sensing, reliable and durable sensors for monitoring diverse human motions. To assess the sensory response performance, the relative resistance changes of the hydrogel sensor under various tensile strains were recorded in Fig. 3g, in which the curve was fitted into a quadratic equation $y=A \varepsilon^{2}+B \varepsilon+C$ (where $y$ is the resistance variation and $\varepsilon$ is the tensile strain). Then, the response sensitivity of the hydrogel sensor could be quantitatively estimated by the GF [34-36]. The average GF of the hydrogel sensor was calculated as 1.2 at a strain of $500 \%$, which highly outperforms multiple reported resistive and capacitive strain sensors (Fig. 3h) [37-46]. The synergistic effects of the high ionic conductivity and mechanical softness of the Al-alginate/PAM hydrogel contributed to the hydrogel sensor's superior sensitivity and electrical stability.

\section{Human motion detection of the super-tough hydrogel sensor}

For monitoring macro-scale human activities in real time, super-tough hydrogels can be designed as wearable stain sensors directly contacted on the skin and joints. For instance, when a hydrogel sensor was attached to the finger, the bending process at different angles could be precisely reflected as the changes in resistance (Fig. 4a). The relative signals on stimulus enhanced as the bending angle increased from $30^{\circ}$ to $90^{\circ}$, and the resistance change ratio $\left(\Delta R / R_{0}\right)$ was constant while the finger was repeatedly bent at a certain angle. Similarly, when the hydrogel sensor was mounted on the wrist and bent to $30^{\circ}, 60^{\circ}$, and $90^{\circ}$, the real-time sensor generated repeatable signals of $\sim 10 \%, 18 \%$, and $26 \%$ (Fig. $4 b$ ).

Real-time motions of the knee joint could be reliably detected on flexing, squatting, jumping, and marching activities (Fig. 4c). The relative resistance variations of the knee-mounted sensor were increased from a standing posture to a flexion state and maintained a constant value with the knee flexed. On squatting down, the sensor attached to the knee joint was stretched further, thus increasing the resistance change ratio. Subsequently, the considerable rise in resistance changes resulted from the rapid knee flexion before the jump, and the drop in resistance changes to the initial value was attributed to the knee extension when the wearer landed and returned to a standing pose. Moreover, when the wearer was marching for a few steps, a repetitive increase and decrease in relative resistance changes originating from consecutive flexion and extension of the knee joint were detected and recorded by the highly sensitive knee-mounted sensor.

When attached to the palm, the hydrogel sensor could produce clear signals with a high signal-to-noise ratio 


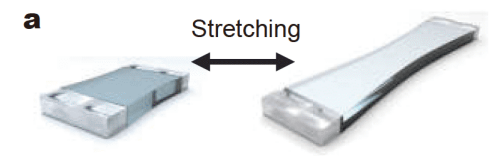

b
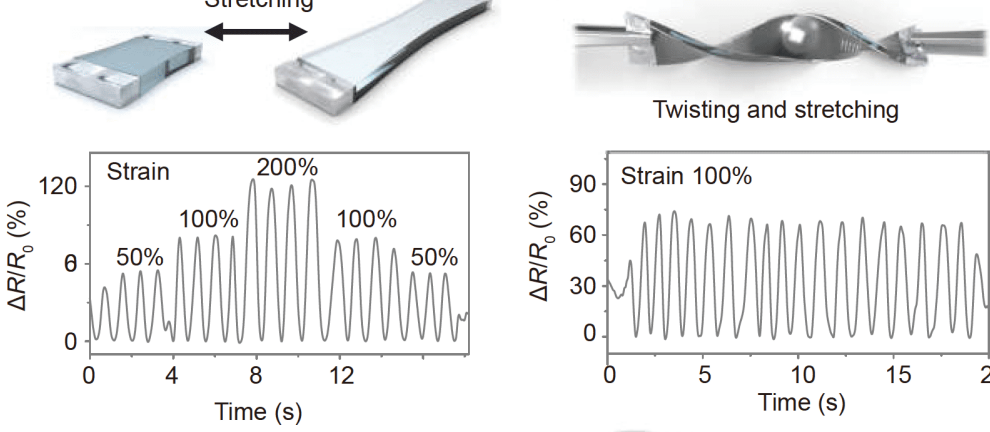

d

Folding and squeezing
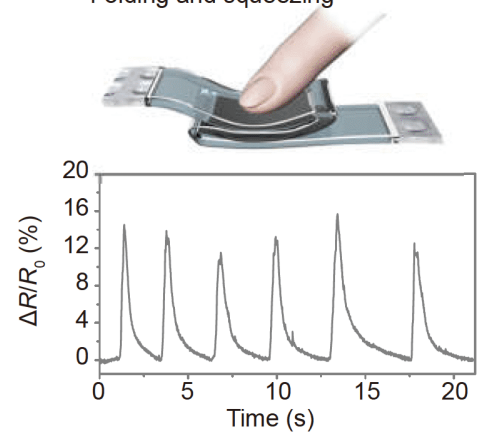

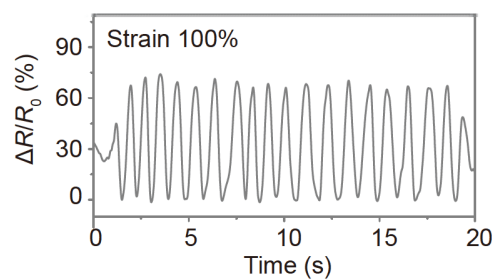

e

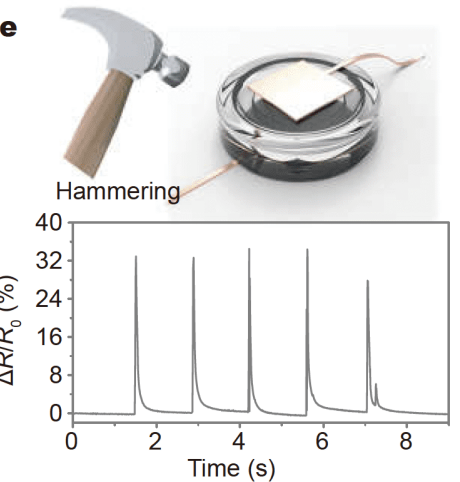

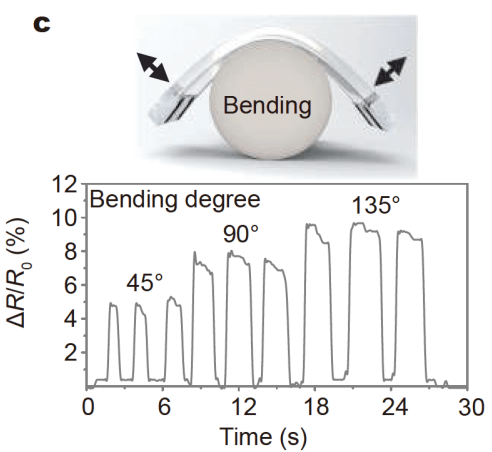

$\mathbf{f}$

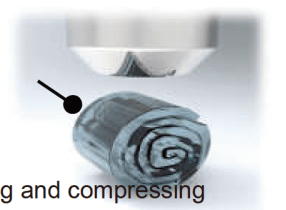

Rolling and compressing

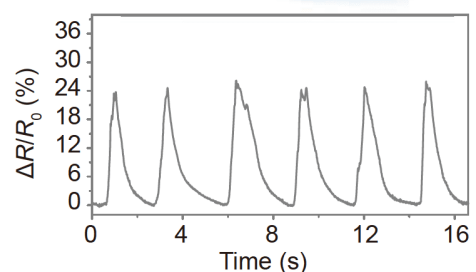

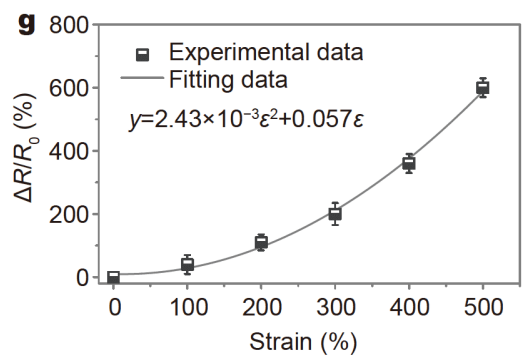

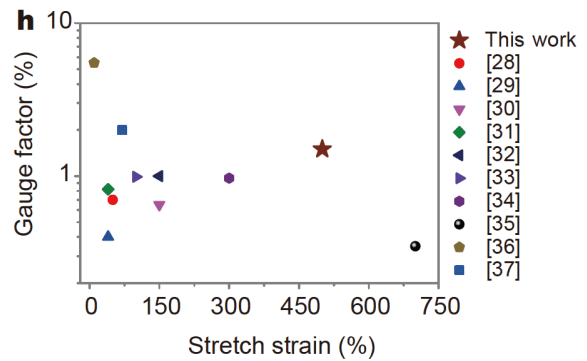

Figure 3 (a) Signals from the hydrogel sensor under different stretch strains (50\%, 100\%, and 200\%). (b) Signals from the hydrogel sensor recorded during stretching to $100 \%$ strain in a twisting state. (c) Signals from the hydrogel sensor recorded at different bending states $\left(45^{\circ}\right.$, $90^{\circ}$, and $\left.135^{\circ}\right)$. (d) Signals from the hydrogel sensor recorded while it was dynamically compressed at folded state. (e) Signals from the hydrogel sensor recorded while being hammered consecutively. (f) Signals from the hydrogel sensor recorded while it was dynamically compressed at rolled state. (g) Profile of the resistance variations versus stretch strain for the Al-alginate/PAM hydrogel sensor. (h) GF comparison as a function of stretch strain of other reported resistive and capacitive sensors.

during consecutive applauding with a maximum resistance change of $\sim 28 \%$ (Fig. $4 \mathrm{~d}$ ). Fig. $4 \mathrm{e}$ shows the sensory response of the super-tough strain sensor attached to the elbow joint. The elbow-mounted sensor could distinguishably respond to different elbow motions and detect resistance changes, in addition to the bending degree variation of the elbow joint. Like the monitoring of the knee joint, the resistance response signal was simultaneously increased as the bending behavior of the elbow joint and remained constant when the elbow was kept in a flexed state. Furthermore, a thin-film strain sensor attached to the protruding part of the throat ex- hibited high-resolution detection to slight vibrations from vocal cords when the wearer normally said "thank you", "hi", and "hello" (Fig. 4f).

With the flexible array sensor comprising aligned super-tough hydrogels, the $2 \mathrm{D}$ distribution of pressure force can be detected, making it possible to develop a $2 \mathrm{D}$ sensing electronic skin [40,47]. Accordingly, a $7 \times 7$ array of hydrogel sensors was designed and fabricated, in which each hydrogel pixel $(4 \mathrm{~mm} \times 5 \mathrm{~mm})$ was connected by copper tapes (Fig. 4g-i and Fig. S14). When the pixels of $\mathrm{B} 5, \mathrm{C} 2, \mathrm{E} 4$, and G3 were pressurized, the resistance change ratio of each pixel was recorded in the computer, 

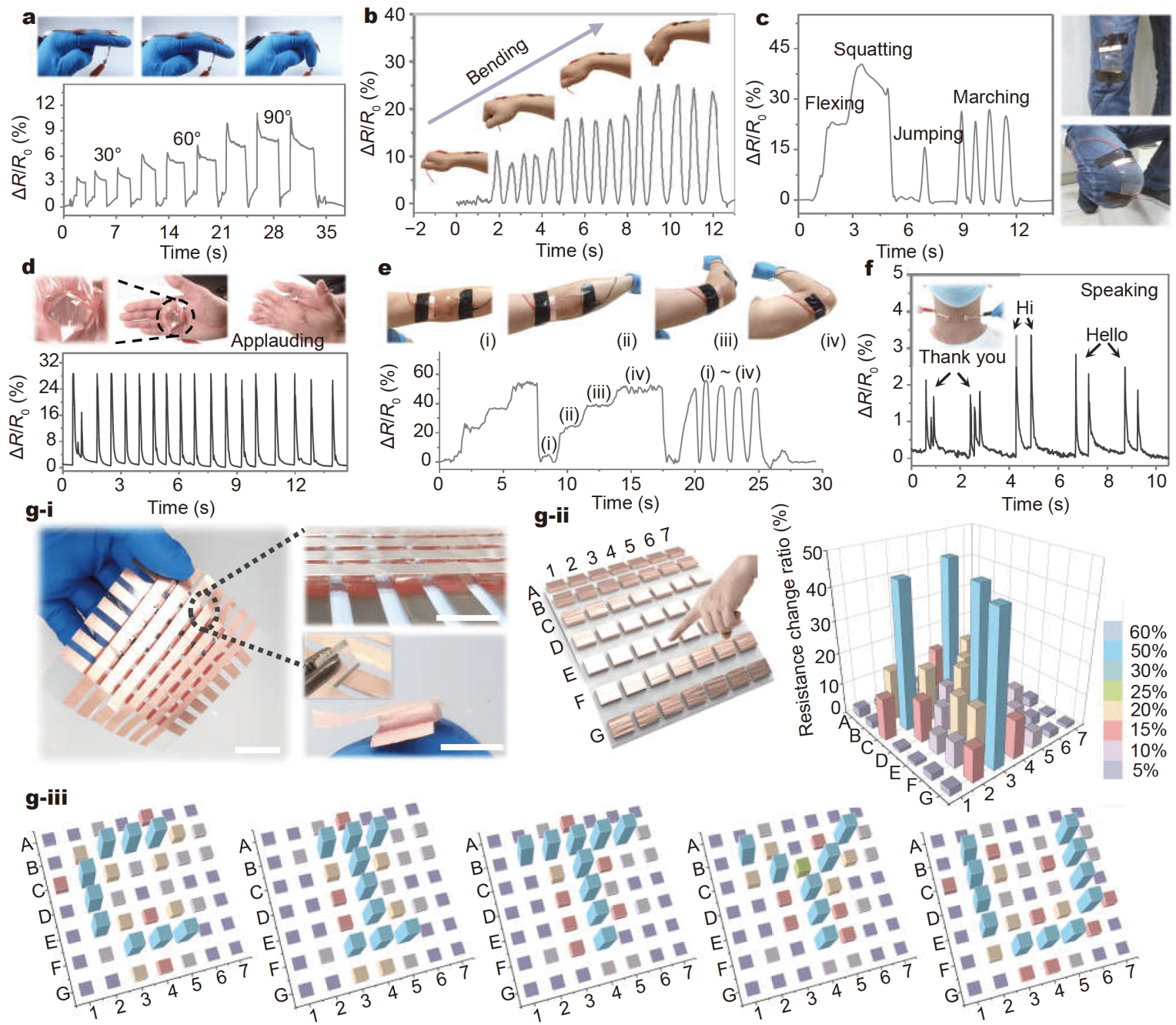

Figure 4 (a) Electrical resistance changes of the hydrogel sensors attached to an index finger as a function of bending angle. (b) Relative resistance responses of hydrogel sensors attached to the wrist under cyclically bending. (c) Signals from the hydrogel sensor under various knee joint motions. (d) Resistance changes of the hydrogel sensors attached to the palms during applauding. (e) Resistance variations of the hydrogel sensor attached to the elbow joint at various bending states. (f) Signals from hydrogel sensors attached to the throat when saying "thank you", "hi", and "hello". (g-i) Digital photos of a flexible $7 \times 7$ array sensor constructed by connecting hydrogel cubes with aligned copper tapes. Each cube serves as an individual pixel. Scale bars: $1 \mathrm{~cm}$. (g-ii) Prism map of the hydrogel array sensor while pressing on B5, C2, E4, and G3 pixels. (g-iii) Electronic logo of "CITYU" produced by finger pressing on the flexible array sensor.

thus producing a sharp signal without crosstalk (Fig. $4 \mathrm{~g}$ ii). With this powerful hydrogel sensor, an electronic CITYU logo was written with a finger-sliding motion (Fig. 4g-iii). For instance, when the finger slid on the array surface from $\mathrm{B} 5$ to $\mathrm{B} 3, \mathrm{C} 2$ to E2, and finally F3 to $\mathrm{F} 5$, an electric signal " $\mathrm{C}$ " was successfully generated. Similarly, electrical signals of other letters were conveniently obtained to accomplish the electronic "CITYU" logo. This sophisticated sensor device demonstrates that it is possible to fabricate next-generation touchpad and eskin with a large array comprising thousands of small pixels [48].

\section{Demonstrations of the super-tough pressure sensor under} severe mechanical stimuli

In addition to the softness that allows arbitrary deformations for strain sensing, the hydrogel sensor possesses ultra-high toughness against severe compression because of the dual-crosslinked structural design with enhancing modulus and fracture energy. To verify this ability, Fig. 5 a shows the compression and recovery of a cylindrical hydrogel sensor. The relative resistance 

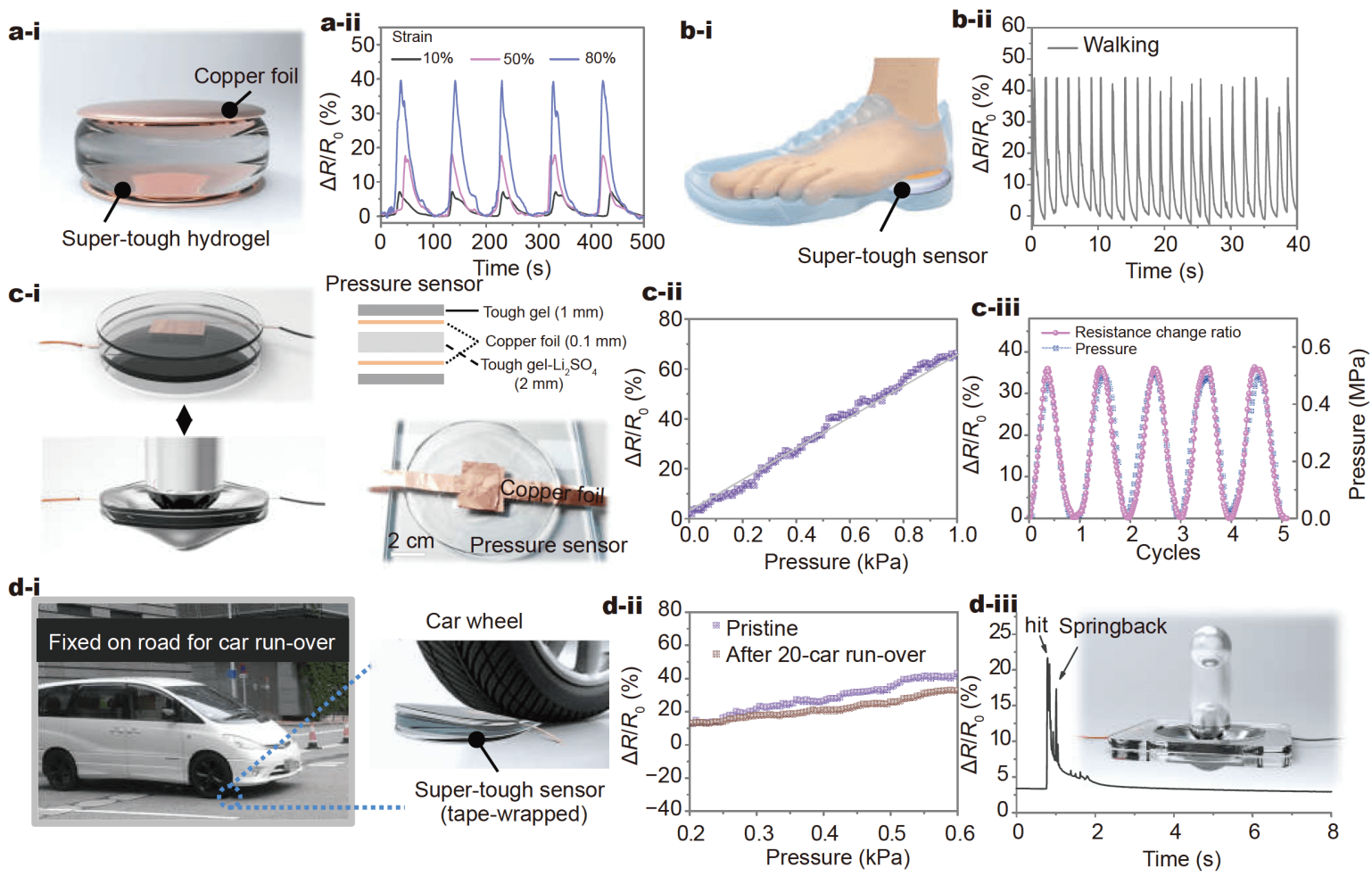

Figure 5 (a) Relative resistance variations of cylindrical hydrogel sensors during five cyclically compressive cycles at diverse strains of $10 \%, 50 \%$, and $80 \%$. (b) Schematic of a hydrogel sensor placed under the foot and the corresponding real-time signals recorded during consecutive walking motions. (c-i) Design of pressure hydrogel sensor and schematic of resistance-compression measurements.(c-ii) Resistance-pressure curve of hydrogel pressure sensor measured in the range of $0-1 \mathrm{kPa}$. (c-iii) Capacitance and pressure of hydrogel pressure sensor cyclically compressed to $0.5 \mathrm{kPa}$ stress. (d-i) Illustration of extremely harsh conditions on hydrogel pressure sensor experiencing car run-over. (d-ii) Capacitance and pressure of a hydrogel pressure sensor during cyclical compression to $0.5 \mathrm{kPa}$ stress tested after being run over by 20 cars. (d-iii) Real-time resistance changes when a metal ball fell onto the hydrogel pressure sensor after being run over by cars.

changes of the hydrogel sensor were recorded during five consecutive cycles at diverse strains of $10 \%, 50 \%, 80 \%$. The hydrogel sensor's response signals did not fluctuate much during cyclic compression, indicating excellent anti-fatigue property against mechanical stress stimulus. Subsequently, we placed a cling-film-wrapped cylindrical hydrogel sensor underfoot and trod on the sensor while walking. The outputs are quantitatively correlated to the continuously treading movement (Fig. 5b).

Furthermore, we used the ionic conductive hydrogel to fabricate a pressure sensor with a radius of $60 \mathrm{~mm}$ (Fig. 5c-i). Note that a piece of ionic conductive hydrogel was placed between two grounded copper stages, and another two hydrogel films were covered on both outer sides to serve as protectors. Subsequently, the relative resistance variations of the pressure sensor were measured using a mechanical testing apparatus by gradually compressing the hydrogel sensor at a strain rate of $0.5 \mathrm{~min}^{-1}$. Although the areal expansion of the pressure sensor was more or less constrained by the surrounding hydrogel protectors when the sensor was compressed in thickness, a significant resistance change response was still observed (Fig. 5c-ii). In this measurement, the pressure was defined as the imposed force divided by the area of the undeformed surface before compression. Then, the relative resistance change of the pressure sensor was recorded as a function of the compressive pressure, which indicates that the resistance variation was roughly linearly responsive as the pressure gradually increased to $1 \mathrm{kPa}$ (Fig. 5c-iii).

Subsequently, as shown in Fig. 5d-i, the pressure sensor was fixed on a road with tape to test its endurance capability under the huge catastrophic impact of car runover. Surprisingly, the comparison profiles of the resistance-compression effect before and after random 20car run-over showed that only a small signal decay was observed in resistance variations during compression (Fig. 5d-ii). The slight reduction in resistance response could be attributed to the wrinkles inside the copper tape electrodes after repeated car run-over, which decreased 
the viscosity of the electrode-electrolyte contact area. Moreover, after exposure to can run-overs, the pressure sensor showed no resistance changes after a 510-g metallic ball fell onto the pressure sensor from a certain height, demonstrating superior durability and high sensitivity (Fig. 5d-iii).

\section{CONCLUSIONS}

In conclusion, a highly soft/flexible yet dynamically super-tough hydrogel sensor was developed based on an energy-dissipative dual-crosslinked hydrogel conductor, which comprises interpenetrating networks of covalently crosslinked PAM and physically $\mathrm{Al}^{3+}$-crosslinked alginate. The dynamically tough hydrogel conductor demonstrated high ionic conductivity $\left(30.2 \mathrm{mS} \mathrm{cm} \mathrm{cm}^{-1}\right)$, ultra-stretchability ( $>600 \%$ strain), and superior linear dependence of strain sensitivity with a maximum GF of 1.2 at $500 \%$ strain. These synergistic effects enabled the hydrogel sensors to display stable and reliable electrical responses under dynamic mechanical stimuli such as repeated stretching, severe squeezing, violent hammering, rolling, and compression. More impressively, a planar pressure sensor could survive extremely harsh conditions, such as successive treading and even being run over by 20 cars on the road. Moreover, the flexible and wearable strain sensor could reliably and quantitatively detect a large range of human motions such as various bending motions of the finger, elbow, wrist, and knee joints, and speaking. For extended application, an array of aligned hydrogel sensors was developed as 2D sensors for detecting tiny force distributions. We believe that the supertough hydrogels promise great inspiration for developing flexible and wearable sensor devices having softness, mechanical durability, and superior sensory capabilities.

Received 9 January 2021; accepted 19 March 2021; published online 21 June 2021

1 Jiang D, Wang Y, Li B, et al. Flexible sandwich structural strain sensor based on silver nanowires decorated with self-healing substrate. Macromol Mater Eng, 2019, 304: 1900074

2 Lu L, Zhou Y, Pan J, et al. Design of helically double-leveled gaps for stretchable fiber strain sensor with ultralow detection limit, broad sensing range, and high repeatability. ACS Appl Mater Interfaces, 2019, 11: 4345-4352

3 Zhai W, Xia Q, Zhou K, et al. Multifunctional flexible carbon black/polydimethylsiloxane piezoresistive sensor with ultrahigh linear range, excellent durability and oil/water separation capability. Chem Eng J, 2019, 372: 373-382

4 Zhou Y, Zhan P, Ren M, et al. Significant stretchability enhancement of a crack-based strain sensor combined with high sensitivity and superior durability for motion monitoring. ACS Appl Mater Interfaces, 2019, 11: 7405-7414
5 Zang Y, Zhang F, Di C, et al. Advances of flexible pressure sensors toward artificial intelligence and health care applications. Mater Horiz, 2015, 2: 140-156

6 Cheng Y, Wang R, Sun J, et al. A stretchable and highly sensitive graphene-based fiber for sensing tensile strain, bending, and torsion. Adv Mater, 2015, 27: 7365-7371

7 Sun $\mathrm{H}$, Zhou K, Yu Y, et al. Highly stretchable, transparent, and bio-friendly strain sensor based on self-recovery ionic-covalent hydrogels for human motion monitoring. Macromol Mater Eng, 2019, 304: 1900227

8 Li X, Zong L, Wu X, et al. Biomimetic engineering of spider silk fibres with graphene for electric devices with humidity and motion sensitivity. J Mater Chem C, 2018, 6: 3212-3219

9 Wang Y, Jia Y, Zhou Y, et al. Ultra-stretchable, sensitive and durable strain sensors based on polydopamine encapsulated carbon nanotubes/elastic bands. J Mater Chem C, 2018, 6: 8160-8170

10 Xu J, Wang S, Wang GJN, et al. Highly stretchable polymer semiconductor films through the nanoconfinement effect. Science, 2017, 355: 59-64

11 Boland CS, Khan U, Ryan G, et al. Sensitive electromechanical sensors using viscoelastic graphene-polymer nanocomposites. Science, 2016, 354: 1257-1260

12 Sekitani T, Noguchi Y, Hata K, et al. A rubberlike stretchable active matrix using elastic conductors. Science, 2008, 321: 1468-1472

13 Dickey MD. Stretchable and soft electronics using liquid metals. Adv Mater, 2017, 29: 1606425

14 Shi L, Zhu T, Gao G, et al. Highly stretchable and transparent ionic conducting elastomers. Nat Commun, 2018, 9: 2630

15 Lei Z, Wu P. A highly transparent and ultra-stretchable conductor with stable conductivity during large deformation. Nat Commun, 2019, 10: 3429

16 Ding Y, Zhang J, Chang L, et al. Preparation of high-performance ionogels with excellent transparency, good mechanical strength, and high conductivity. Adv Mater, 2017, 29: 1704253

17 Chen B, Lu JJ, Yang CH, et al. Highly stretchable and transparent ionogels as nonvolatile conductors for dielectric elastomer transducers. ACS Appl Mater Interfaces, 2014, 6: 7840-7845

18 Cao Y, Morrissey TG, Acome E, et al. A transparent, self-healing, highly stretchable ionic conductor. Adv Mater, 2017, 29: 1605099

19 Lei Z, Wang Q, Sun S, et al. A bioinspired mineral hydrogel as a self-healable, mechanically adaptable ionic skin for highly sensitive pressure sensing. Adv Mater, 2017, 29: 1700321

20 Dong K, Wang YC, Deng J, et al. A highly stretchable and washable all-yarn-based self-charging knitting power textile composed of fiber triboelectric nanogenerators and supercapacitors. ACS Nano, 2017, 11: 9490-9499

21 Liu Z, Wang D, Tang Z, et al. A mechanically durable and devicelevel tough $\mathrm{Zn}-\mathrm{MnO}_{2}$ battery with high flexibility. Energy Storage Mater, 2019, 23: 636-645

22 Baumberger T, Ronsin O. Cooperative effect of stress and ion displacement on the dynamics of cross-link unzipping and rupture of alginate gels. Biomacromolecules, 2010, 11: 1571-1578

23 Kong HJ, Wong E, Mooney DJ. Independent control of rigidity and toughness of polymeric hydrogels. Macromolecules, 2003, 36: $4582-4588$

24 Sun JY, Zhao X, Illeperuma WRK, et al. Highly stretchable and tough hydrogels. Nature, 2012, 489: 133-136

25 Rivlin RS, Thomas AG. Rupture of rubber. I. Characteristic energy for tearing. J Polym Sci, 1953, 10: 291-318

26 Guan L, Yan S, Liu X, et al. Wearable strain sensors based on 
casein-driven tough, adhesive and anti-freezing hydrogels for monitoring human-motion. J Mater Chem B, 2019, 7: 5230-5236

27 Sakai T, Akagi Y, Kondo S, et al. Experimental verification of fracture mechanism for polymer gels with controlled network structure. Soft Matter, 2014, 10: 6658-6665

28 Lake GJ, Thomas AG. The strength of highly elastic materials. Proc R Soc Lond A, 1967, 300: 108-119

29 Matsuda T, Nakajima T, Fukuda Y, et al. Yielding criteria of double network hydrogels. Macromolecules, 2016, 49: 1865-1872

30 Liao H, Guo X, Wan P, et al. Conductive MXene nanocomposite organohydrogel for flexible, healable, low-temperature tolerant strain sensors. Adv Funct Mater, 2019, 29: 1904507

31 Guo Y, Zhong M, Fang Z, et al. A wearable transient pressure sensor made with MXene nanosheets for sensitive broad-range human-machine interfacing. Nano Lett, 2019, 19: 1143-1150

32 Ma Z, Shi W, Yan K, et al. Doping engineering of conductive polymer hydrogels and their application in advanced sensor technologies. Chem Sci, 2019, 10: 6232-6244

33 Guo Y, Bae J, Fang Z, et al. Hydrogels and hydrogel-derived materials for energy and water sustainability. Chem Rev, 2020, 120: 7642-7707

34 Cai G, Wang J, Qian K, et al. Extremely stretchable strain sensors based on conductive self-healing dynamic cross-links hydrogels for human-motion detection. Adv Sci, 2017, 4: 1600190

35 Jian M, Wang C, Wang Q, et al. Advanced carbon materials for flexible and wearable sensors. Sci China Mater, 2017, 60: 10261062

36 Li S, Zhang Y, Wang Y, et al. Physical sensors for skin-inspired electronics. InfoMat, 2020, 2: 184-211

37 Yao S, Zhu Y. Wearable multifunctional sensors using printed stretchable conductors made of silver nanowires. Nanoscale, 2014, 6: $2345-2352$

38 Lipomi DJ, Vosgueritchian M, Tee BCK, et al. Skin-like pressure and strain sensors based on transparent elastic films of carbon nanotubes. Nat Nanotech, 2011, 6: 788-792

39 Wang Z, Huang Y, Sun J, et al. Polyurethane/cotton/carbon nanotubes core-spun yarn as high reliability stretchable strain sensor for human motion detection. ACS Appl Mater Interfaces, 2016, 8: 24837-24843

40 Yamada T, Hayamizu Y, Yamamoto Y, et al. A stretchable carbon nanotube strain sensor for human-motion detection. Nat Nanotech, 2011, 6: 296-301

41 Shin UH, Jeong DW, Park SM, et al. Highly stretchable conductors and piezocapacitive strain gauges based on simple contact-transfer patterning of carbon nanotube forests. Carbon, 2014, 80: 396-404

42 Yuk H, Zhang T, Parada GA, et al. Skin-inspired hydrogel-elastomer hybrids with robust interfaces and functional microstructures. Nat Commun, 2016, 7: 12028

43 Cai L, Song L, Luan P, et al. Super-stretchable, transparent carbon nanotube-based capacitive strain sensors for human motion detection. Sci Rep, 2013, 3: 3048

44 Frutiger A, Muth JT, Vogt DM, et al. Capacitive soft strain sensors via multicore-shell fiber printing. Adv Mater, 2015, 27: 2440-2446

45 Kong JH, Jang NS, Kim SH, et al. Simple and rapid micropatterning of conductive carbon composites and its application to elastic strain sensors. Carbon, 2014, 77: 199-207

46 Amjadi M, Pichitpajongkit A, Lee S, et al. Highly stretchable and sensitive strain sensor based on silver nanowire-elastomer nanocomposite. ACS Nano, 2014, 8: 5154-5163

47 Wang Z, Chen J, Cong Y, et al. Ultrastretchable strain sensors and arrays with high sensitivity and linearity based on super tough conductive hydrogels. Chem Mater, 2018, 30: 8062-8069

48 Wang S, Xu J, Wang W, et al. Skin electronics from scalable fabrication of an intrinsically stretchable transistor array. Nature, 2018, 555: 83-88

Acknowledgements This work was financially supported by the Science Technology and Innovation Committee of Shenzhen Municipality under Shenzhen Technology Project (JSGG20180508151728414), and the Department of Science and Technology of Guangdong Province under Guangdong Science and Technology Project (2018B020208001).

Author contributions Mo F and Wang $\mathrm{Z}$ conducted the experiment; Li Q and Lv S did some characterizations; Gai W performed some data analysis and offered helpful suggestions; Jiang $\mathrm{R}$ and Zhi $\mathrm{C}$ designed this study, analyzed the data, and wrote the paper. All authors contributed to the general discussion.

Conflict of interest There are no conflicts of interest to declare.

Supplementary information Experimental details and supporting data are available in the online version of the paper.

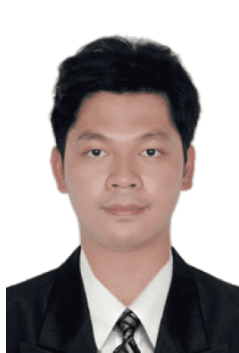

Funian Mo received his $\mathrm{PhD}$ degree from the City University of Hong Kong, China, under the supervision of Prof. Chunyi Zhi. He is currently an assistant researcher at the Flexible Printed Electronics Technology Center at Harbin Institute of Technology (Shenzhen), China. His research interests cover the areas of polymer chemistry, nanotechnology, and flexible energy storage devices with a focus on $\mathrm{Zn}$-ion batteries.

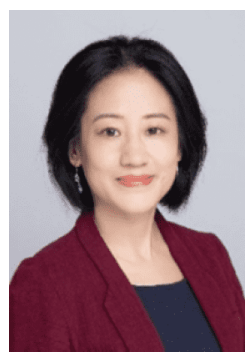

Ruijuan Jiang obtained her $\mathrm{PhD}$ degree in bridge and structure engineering from the Department of Civil Engineering, University of Hong Kong. After two years' postdoc at the University of Hong Kong, she joined Shenzhen Municipal Engineering Design and Research Institute Co., Ltd, as an engineer engaged in bridge engineering research and design. Now, Dr. Jiang is a professorate senior engineer and has extensive experience in structural health monitoring, engineering design, and research of

composite bridges.

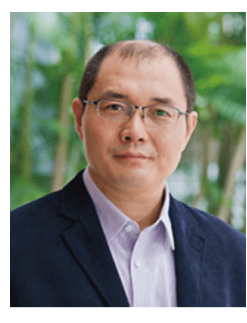

Chunyi Zhi obtained his $\mathrm{PhD}$ degree in condensed matter physics from the Institute of Physics, Chinese Academy of Sciences. After two years' postdoc at the National Institute for Materials Science (NIMS) in Japan, he was promoted to be a researcher of the International Center for Young Scientists (ICYS) program, researcher (faculty) and senior researcher (permanent position) at the NIMS. Dr. Zhi is now a professor at the Department of Materials Science and Engineering (MSE), City University of Hong

Kong. Dr. Zhi has extensive experience in sensors, flexible energy storage, aqueous electrolyte batteries, and $\mathrm{Zn}$-ion batteries. 


\section{基于能量耗散型双交联水凝胶的超㓞性动态传感器}

莫富年 ${ }^{1,2}$, 王子峰 ${ }^{2}$, 姜瑞娟 $^{*}$, 盖卫明 ${ }^{3}$, 李清 $^{2}$, 吕双坤 ${ }^{4}$, 支春义 $2^{2^{*}}$

摘要 本征可拉伸导体在电子皮肤和柔性可穿戴传感器领域中正 在快速发展. 然而, 由于电子-机械性能和传感性能难以完美结合, 人造皮肤材料/器件的实际应用尚未实现. 一方面是由于传统的水 凝胶导体中掺杂的无机导电剂通常与周围的弹性网络不相容, 从 而导致机械强度降低. 另一方面由于水凝胶通常受到严重的机械 撞击或较大的变形, 特别是当缺口出现时, 容易受到损坏. 本研究 中, 我们基于具有耗散能力的双交联导电水凝胶, 开发出一种机械 强度高且柔韧性好的应变传感器. 具有动态韧性的水凝胶导体显 示出较高的离子电导率 $\left(30.2 \mathrm{mS} \mathrm{cm}^{-1}\right.$ ), 超长的拉伸性( $>600 \%$ 应 变)和优异的线性正相关的应变敏感性, 在 $500 \%$ 应变下其最大应变 灵敏因数为 1.2 . 基于这些协同作用, 制备的水凝胶应变传感器能够 可靠稳定地检测人体的大幅度运动和小幅度动作的微小振动. 此 外, 它还具有超强的柔韧性, 可以承受连续的踩踏压力, 甚至在道 路上被行驶的车辆碾压 20 次后仍能保持正常运行. 这些表征证实 了其卓越的机械耐久性和可靠性, 在开发下一代实用性传感器方 面显示出巨大潜力. 